\title{
Editorial
}

Falk Schreiber*, Padraig Gleeson, Martin Golebiewski, Thomas E. Gorochowski, Michael Hucka, Sarah M. Keating, Matthias König, Chris J. Myers, David P. Nickerson, Björn Sommer and Dagmar Waltemath

\section{Specifications of standards in systems and synthetic biology: status and developments in 2021}

https://doi.org/10.1515/jib-2021-0026

\begin{abstract}
This special issue of the Journal of Integrative Bioinformatics contains updated specifications of COMBINE standards in systems and synthetic biology. The 2021 special issue presents four updates of standards: Synthetic Biology Open Language Visual Version 2.3, Synthetic Biology Open Language Visual Version 3.0, Simulation Experiment Description Markup Language Level 1 Version 4, and OMEX Metadata specification Version 1.2. This document can also be consulted to identify the latest specifications of all COMBINE standards.
\end{abstract}

\section{Introduction}

COMBINE ('COmputational Modeling in BIology' NEtwork) [1, 2] is the formal entity coordinating the development of standards in systems and synthetic biology. It was founded in 2009, and since then supports and coordinates standard developments across the globe and thereby fosters and moderates discussions; designs and implements dissemination strategies; and offers a central access point to specifications and library support. The COMBINE coordination board organises two annual community meetings - the COMBINE forum and the HARMONY hackathon.

Falk Schreiber, Department of Computer and Information Science, University of Konstanz, Konstanz, Germany; and Faculty of Information Technology, Monash University, Melbourne, Australia, E-mail: falk.schreiber@uni-konstanz.de

Padraig Gleeson and Sarah M. Keating, University College London, London, UK, E-mail: p.gleeson@ucl.ac.uk (P. Gleeson), s.keating@ucl.ac.uk (S. M. Keating)

Martin Golebiewski, Heidelberg Institute for Theoretical Studies (HITS), Heidelberg, Germany,

E-mail: martin.golebiewski@h-its.org

Thomas E. Gorochowski, School of Biological Sciences, University of Bristol, Bristol, UK,

E-mail: thomas.gorochowski@bristol.ac.uk

Michael Hucka, California Institute of Technology, Pasadena, USA, E-mail: mhucka@caltech.edu

Matthias König, Institute for Theoretical Biology, Humboldt-University Berlin, Berlin, Germany,

E-mail: konigmatt@googlemail.com.https://orcid.org/0000-0003-1725-179X

Chris J. Myers, Department of Electrical, Computer, and Energy Eng., University of Colorado Boulder, Boulder, USA,

E-mail: Chris.Myers@colorado.edu

David P. Nickerson, Auckland Bioengineering Institute, University of Auckland, Auckland, New Zealand,

E-mail: david.nickerson@gmail.com

Björn Sommer, Royal College of Arts, London, UK, E-mail: bjorn.sommer@rca.ac.uk

Dagmar Waltemath, University Medicine Greifswald, Rostock, Germany, E-mail: dagmar.waltemath@uni-greifswald.de.

https://orcid.org/0000-0002-5886-5563

Ә Open Access. @ 2021 Falk Schreiber et al., published by De Gruyter. (c) $)_{\text {BY }}$ 4.0 International License. 


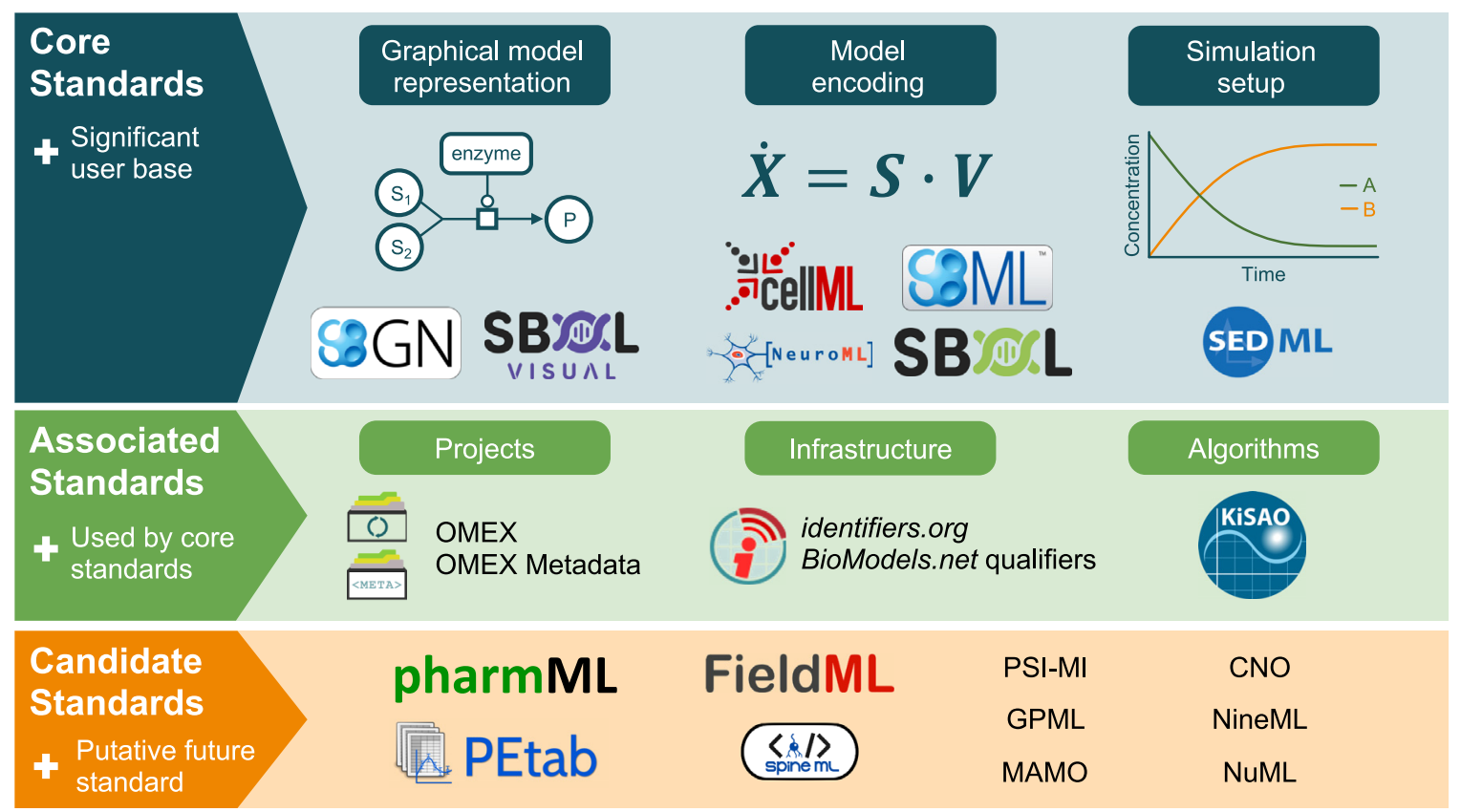

Figure 1: COMBINE standards and associated efforts (updated from [3]).

Figure 1 shows an overview of COMBINE standards and associated initiatives. This editorial will present the latest specifications of all COMBINE standards, and this special issue highlights updates over the last year, namely the releases of the following specifications: OMEX Metadata Version 1.2, SED-ML Level 1 Version 4, SBOL Visual 2.3 and 3.0. Special issues on COMBINE standards have been published since 2016, and earlier editions [3-7] provide updates for the years 2015-2020.

The community websites of the different standards and initiatives are available from the COMBINE web site at https://co.mbine.org/. This web site also contains links to COMBINE events such as the COMBINE forum and the HARMONY hackathon. Additional information, the history of COMBINE as well as its organisation can be found in publications, for example, by Hucka et al. [2], Myers et al. [8], Waltemath et al. [9] or Dräger and Waltemath [10].

This editorial will in the remaining part provide a brief updated overview of the latest specifications of the COMBINE standards and related initiatives (updated from [7]).

\section{Current versions of COMBINE standards}

Please refer to the following (most up-to-date) specifications when using COMBINE standards. New specifications or updates of existing specifications are highlighted with NEW.

\subsection{Core standards}

\subsubsection{BioPAX (Biological PAthway eXchange)}

BioPAX is a standard language for integration, exchange and analysis of biological pathway data. It is expressed in OWL. The current specification is: 


\begin{tabular}{lll}
\hline Standard & Specification & Reference \\
\hline BioPAX [11] & BioPAX & {$[12]$} \\
\hline
\end{tabular}

\subsubsection{CellML}

The CellML language is an XML markup language to store and exchange computer-based mathematical models. The current specifications are:

\begin{tabular}{lll}
\hline Standard & Specification & Reference \\
\hline CellML [13] & CellML 2.0 & {$[14]$} \\
& CellML Metadata Framework 2.0 & {$[15]$} \\
& CellML 1.1 & {$[16]$} \\
\hline
\end{tabular}

\subsubsection{NeuroML}

The neural open markup language (NeuroML) is an XML-based description language that provides a common data format for defining and exchanging descriptions of neuronal cell and network models. The current specification is:

\begin{tabular}{lll}
\hline Standard & Specification & Reference \\
\hline NeuroML $[17,18]$ & NeuroML version 2.1 & {$[17]$} \\
\hline
\end{tabular}

\subsubsection{SBGN (Systems Biology Graphical Notation)}

The systems biology graphical notation (SBGN), is a set standard graphical languages to describe visually biological knowledge. It consists of three languages describing Process Descriptions, Entity Relationships and Activity Flows. In addition, SBGN-ML is an XML-based file format describing the geometry of SBGN maps, while preserving their underlying biological meaning. The current specifications are:

\begin{tabular}{lll}
\hline Standard & Specification & Reference \\
\hline SBGN [19] & SBGN Process Description Level 1 Version 2 & {$[20]$} \\
& SBGN Entity Relationship Level 1 Version 2.0 & {$[21]$} \\
& SBGN Activity Flow Level 1 Version 1.2 & {$[22]$} \\
& SBGN Markup Language Version 0.3 & {$[23]$} \\
\hline
\end{tabular}

\subsubsection{SBML (Systems Biology Markup Language)}

The systems biology markup language (SBML) is a computer-readable XML format for representing models of biological processes. SBML is suitable for, but not limited to, models using a process description approach. SBML development is coordinated by an elected editorial board and central developer team. The current specifications are: 


\begin{tabular}{llr}
\hline Standard & Specification & Reference \\
\hline SBML [24] & SBML Level 3 Core, Version 2 Release 2 & {$[25]$} \\
& SBML Level 3 Package: Distributions, Version 1, Release 1 & {$[26]$} \\
& SBML Level 3 Package: Flux Balance Constraints Version 2 & {$[27]$} \\
SBML Level 3 Package: Groups, Version 1 & {$[28]$} & {$[29]$} \\
SBML Level 3 Package: Hierarchical Model Composition, Version 1 & {$[30]$} \\
SBML Level 3 Package: Layout, Version 1 & {$[31]$} \\
SBML Level 3 Package: Multistate, Multicomponent and Multicompartment & {$[32]$} \\
Species, Version 1, Release 2 & {$[33]$} \\
\hline
\end{tabular}

\subsubsection{SBOL (Synthetic Biology Open Language)}

The synthetic biology open language (SBOL) is a language for the description and the exchange of synthetic biological parts, devices, and systems. SBOL visual (SBOLv) is a complementary standard that provides a standard set of glyphs and rules for drawing genetic circuit diagrams. The current specifications are listed in the following table.

NEW Synthetic biology open language visual (SBOL Visual) Version 2.3 [34] updates SBOL visual version 2.2 with several new features. These include higher-level "interactions with interactions", the representation of the binding with a nucleic acid backbone using overlapping glyphs, and a new "unspecified interaction" glyph. In this version, the "insulator" glyph is deprecated and replaced with a new "inert DNA spacer" glyph, and the polypeptide region glyph is now recommended for showing $2 \mathrm{~A}$ sequences.

NEW SBOL Visual Version 3.0 [35] is major revision of the SBOL Visual standard in which the diagrams and glyphs are now defined with respect to the SBOL 3 data model rather than the SBOL 2 data model. In addition, the use of dashed undirected lines for subsystem mappings has been removed, and deprecated material has been removed from the collection of glyphs. Finally, the deprecated BioPAX alternatives to SBO terms have been removed.

\begin{tabular}{lll}
\hline Standard & Specification & Reference \\
\hline SBOL [36] & SBOL Version 3.0.0 & {$[37]$} \\
& SBOL Visual Version 2.3 & {$[34]$} \\
& SBOL Visual Version 3.0 & {$[35]$} \\
\hline
\end{tabular}

\subsubsection{SED-ML (Simulation Experiment Description Markup Language)}

The simulation experiment description markup language is an XML-based format for encoding simulation experiments. SED-ML allows to define the models to use, the experimental tasks to run and which results to produce. SED-ML can be used with models encoded in several languages. The current specification is listed in the following table.

NEW The simulation experiment description markup language (SED-ML) Level 1 Version 4 [38] clarifies previous versions of SED-ML and extends the language with multiple new features. Specifically, enhancements introduced in SED-ML Level 1 Version 4 include (1) enriched plotting capabilities, (2) dimension reductions and math on multidimensional data, (3) support for simple parameter fitting experiments, and (4) a generic Analysis task. Further refinements in this version of the specification aim to clarify the use of SED-ML with non-XML model description formats. 


\begin{tabular}{lll}
\hline Standard & Specification & Reference \\
\hline SED-ML [39] & SED-ML Level 1 Version 4 & {$[38]$} \\
\hline
\end{tabular}

\subsection{Associated standards}

Associated standards provide an additional layer of semantics to COMBINE representation formats. The current specifications are:

\begin{tabular}{lll}
\hline Standard & Specification & Reference \\
\hline COMBINE Archive [40] & COMBINE Archive 1.0 & {$[41]$} \\
OMEX Metadata & OMEX Metadata Version 1.2 & {$[42]$} \\
BioModels.net qualifiers [43] & - & {$[44]$} \\
Identifiers.org URIs [45] & - & {$[46]$} \\
Systems Biology Ontology [47] & [external] Bioportal & {$[48]$} \\
Kinetic Simulation Algorithm Ontology [47] & [external] Bioportal & {$[49]$} \\
\hline
\end{tabular}

A COMBINE archive is a single file bundling the various documents and all relevant information necessary for a modelling and simulation project. The archive is encoded using the Open Modeling EXchange format (OMEX).

COMBINE archive metadata provides a harmonised, community-driven approach for annotating a variety of standardised model and data representation formats within a COMBINE archive.

BioModels.net qualifiers are standardised relationships (predicates) that specify the relation between an object represented in a description language and the external resource used to annotate it. MIRIAM Unique Resource Identifiers allow one to uniquely and unambiguously identify an entity in a stable and perennial manner. MIRIAM Registry is a set of services and resources that provide support for generating, interpreting and resolving MIRIAM URIs. Through the Identifiers.org technology, MIRIAM URIs can be dereferenced in a flexible and robust way. MIRIAM URIs are used by SBML, SED-ML, CellML and BioPAX controlled annotation schemes.

The systems biology ontology (SBO) is a set of controlled, relational vocabularies of terms commonly used in systems biology, and in particular in computational modelling. Each element of an SBML file carries an optional attribute sboTerm which value must be a term from SBO. Each symbol of SBGN is associated with an SBO term.

The kinetic simulation algorithm ontology (KiSAO) describes existing algorithms and their interrelationships through their characteristics and parameters. KiSAO is used in SED-ML, which allows simulation software to automatically choose the best algorithm available to perform a simulation and unambiguously refer to it.

The OMEX Metadata Specification is a technical implementation of the community consensus across COMBINE standards to harmonise the way we describe computational models and other resources with metadata [50].

NEW The OMEX Metadata Specification Version 1.2 [42] clarifies the OMEX Metadata Specification 1.0 [51]. Specifically, the main changes introduced in OMEX Metadata Specification Version 1.2 include (1) clarification of authorship and provenance information, (2) clarification of distinction between document- and model-level annotations, and (3) the introduction of an archive-level namespacing convention to ensure reproducible sharing and interpretation of the knowledge graph encoded in the annotations.

Acknowledgements: We thank all members of the COMBINE initiative for their help. DPN is supported by the National Institutes of Health [P41EB023912]. MG is funded by the European Union Horizon2020 framework programme of the European Commission Directorate-General for Research and Innovation (grant 825843) 
as part of the EU-STANDS4PM project (European standardization framework for data integration and datadriven in silico models for personalised medicine), as well as by the Deutsche Forschungsgemeinschaft (DFG, German Research Foundation, grant NFDI 13/1) as part of the German Research Data Infrastructure Consortium NFDI4Health (www.nfdi4health.de). MK and MG are supported by the Federal Ministry of Education and Research (BMBF, Germany) within the research network Systems Medicine of the Liver (LiSyM, grant numbers 031L0054 and 031L0056). MK is also supported by the DFG within the Research Unit Programme FOR 5151 "QuaLiPerF (Quantifying Liver Perfusion-Function Relationship in Complex Resection--A Systems Medicine Approach)” by grant 436883643.

Author contribution: All authors have accepted responsibility for the entire content of this manuscript and approved its submission.

Research funding: None declared.

Conflict of interest statement: Authors state no conflict of interest.

\section{References}

1. Waltemath D, Golebiewski M, Blinov ML, Gleeson P, Hermjakob H, Hucka M, et al. The first 10 years of the international coordination network for standards in systems and synthetic biology (COMBINE). J Integr Bioinform 2020;17:5.

2. Hucka M, Nickerson DP, Bader GD, Bergmann FT, Jonathan C, Demir E, et al. Promoting coordinated development of community-based information standards for modeling in biology: the COMBINE initiative. Front Bioeng Biotechnol 2015;3:19.

3. Schreiber F, Bader GD, Gleeson P, Golebiewski M, Hucka M, Keating SM, et al. Specifications of standards in systems and synthetic biology: status and developments in 2018. J Integr Bioinform 2018;15:13.

4. Schreiber F, Bader GD, Golebiewski M, Hucka M, Kormeier B, Le Novère N, et al. Specifications of standards in systems and synthetic biology. J Integr Bioinform 2015;12:258.

5. Schreiber F, Bader GD, Gleeson P, Golebiewski M, Hucka M, Novère NL, et al. Specifications of standards in systems and synthetic biology: status and developments in 2016. J Integr Bioinform 2016;13:289.

6. Schreiber F, Sommer B, Bader GD, Gleeson P, Golebiewski M, Hucka M, et al. Specifications of standards in systems and synthetic biology: status and developments in 2019. J Integr Bioinform 2019;16:35.

7. Schreiber F, Sommer B, Czauderna T, Golebiewski M, Gorochowski TE, Hucka M, et al. Specifications of standards in systems and synthetic biology: status and developments in 2020. J Integr Bioinform 2020;17:22.

8. Myers C, Bader GD, Gleeson P, Golebiewski M, Hucka M, Le Novère N, et al. A brief history of COMBINE. In: Proceedings of the 2017 winter simulation conference; 2017:884-95 pp.

9. Waltemath D, Bergmann FT, Chaouiya C, Tobias C, Gleeson P, Goble C, et al. Meeting report from the fourth meeting of the computational modeling in biology network (COMBINE). Stand Genom Sci 2014;9:1285-301.

10. Dräger A, Waltemath D. Overview: standards for modeling in systems medicine. In: Systems medicine 1st edition integrative, qualitative and computational approaches, vol 1. Oxford: Academic Press; 2020:345-53 pp.

11. Demir E, Cary MP, Paley S, Fukuda K, Lemer C, Vastrik I, et al. The BioPAX community standard for pathway data sharing. Nat Biotechnol 2010;28:935-42.

12. BioPax. 2017. Available from: http://www.biopax.org/.

13. Cuellar AA, Lloyd CM, Nielsen PF, Bullivant D, Nickerson D, Hunter P. An overview of CellML 1.1, a biological model description language. Simulation 2003;79:740-7.

14. Clerx M, Cooling MT, Cooper J, Garny A, Moyle K, Nickerson DP, et al. CellML 2.0. J Integr Bioinform 2020;17:21.

15. Cooling MT, Hunter PJ. The CellML metadata framework 2.0 specification. J Integr Bioinform 2015;12:260.

16. Cuellar AA, Hedley W, Nelson M, Lloyd CM, Halstead MDB, Bullivant DP, et al. The CellML 1.1 specification. J Integr Bioinform 2015;12:259.

17. Cannon RC, Gleeson P, Crook S, Ganapathy G, Marin B, Piasini E, et al. LEMS: a language for expressing complex biological models in concise and hierarchical form and its use in underpinning NeuroML 2. Front Neuroinf 2014;8:79.

18. Gleeson P, Crook S, Cannon RC, Hines ML, Billings GO, Farinella M, et al. NeuroML: a language for describing data driven models of neurons and networks with a high degree of biological detail. PLoS Comput Biol 2010;6: e1000815. https://doi .org/10.1371/journal.pcbi.1000815.

19. Le Novère N, Hucka M, Mi H, Moodie S, Schreiber F, Sorokin A, et al. The systems biology graphical notation. Nat Biotechnol 2009;27:735-41. 
20. Rougny A, Tourè V, Moodie S, Balaur I, Czauderna T, Borlinghaus H, et al. Systems biology graphical notation: process description language level 1 version 2. J Integr Bioinform 2019;16:22.

21. Sorokin AA, Le Novère N, Luna A, Czauderna T, Demir E, Haw R, et al. Systems biology graphical notation: entity relationship language level 1 version 2. J Integr Bioinform 2015;12:264.

22. Mi H, Schreiber F, Moodie SL, Czauderna T, Demir E, Haw R, et al. Systems biology graphical notation: Activity flow language level 1 version 1.2. J Integr Bioinform 2015;12:265.

23. Bergmann FT, Czauderna T, Dogrusoz U, Rougny A, Dräger A, Toure V, et al. Systems biology graphical notation markup language (SBGNML) version 0.3. J Integr Bioinform 2020;17:16.

24. Hucka M, Finney A, Sauro HM, Bolouri H, Doyle JC, Kitano H, et al. The Systems Biology Markup Language (SBML): a medium for representation and exchange of biochemical network models. Bioinformatics 2003;19:524-31.

25. Hucka M, Bergmann FT, Chaouiya C, Dräger A, Hoops S, Keating SM, et al. The systems biology markup language (SBML): language specification for level 3 version 2 core release 2. J Integr Bioinform 2019;16:21.

26. Smith LP, Moodie SL, Bergmann FT, Gillespie C, Keating SM, König M, et al. SBML level 3 package: distributions, version 1 , release 1. J Integr Bioinform 2020;17:18.

27. Olivier BG, Bergmann FT. SBML level 3 package: flux balance constraints, version 2. J Integr Bioinform 2018;15:82.

28. Hucka M, Smith LP. The systems biology markup language (SBML) level 3 package: groups, version 1 release 1. J Integr Bioinform 2016;13:290.

29. Smith LP, Hucka M, Hoops S, Finney A, Ginkel M, Myers CJ, et al. SBML level 3 package: hierarchical model composition, version 1 release 3. J Integr Bioinform 2015;12:268.

30. Gauges R, Rost U, Sahle S, Wengler K, Bergmann FT. The systems biology markup language (SBML) level 3 package: layout, version 1 core. J Integr Bioinform 2015;12:267.

31. Zhang F, Smith LP, Blinov ML, Faeder J, Hlavacek WS, Tapia JJ, et al. SBML level 3 package: multistate, multicomponent and multicompartment species, version 1, release 2. J Integr Bioinform 2020;17:15.

32. Chaouiya C, Keating SM, Bérenguier D, Naldi A, Thieffry D, van lersel MP, et al. The systems biology markup language (SBML) level 3 package: qualitative models, version 1, release 1. J Integr Bioinform 2015;12:270.

33. Bergmann FT, Keating SM, Gauges R, Sahle S, Wengler K. SBML level 3 package: render, version 1, release 1. J Integr Bioinform 2018;15:78.

34. Baig H, Fontanarossa P, Kulkarni V, McLaughlin J, Vaidyanathan P, Bartley B, et al. Synthetic biology Open Language visual (SBOL visual) version 2.3. J Integr Bioinform 2021;18:2020-0045.

35. Beal J, Baig H, Fontanarrosa P, McLaughlin JA, Scott-Brown J, Vaidyanathan P, et al. Synthetic biology Open Language visual (SBOL visual) version 3.0. J Integr Bioinform 2021;18:2021-0013.

36. Galdzicki M, Clancy KP, Oberortner E, Pocock M, Jacqueline YQ, Cesar AR, et al. The Synthetic Biology Open Language (SBOL) provides a community standard for communicating designs in synthetic biology. Nat Biotechnol 2014;32:545-50.

37. Baig H, Fontanarrosa P, Kulkarni V, McLaughlin JA, Vaidyanathan P, Bartley B, et al. Synthetic biology Open Language (SBOL) version 3.0.0. J Integr Bioinform 2020;17:17.

38. Smith L, Bergmann F, Garny A, Helikar T, Karr J, Nickerson D, et al. Simulation experiment description markup language (SED-ML): level 1 version 4. J Integr Bioinform 2021;18:2021-0021.

39. Waltemath D, Adams R, Bergmann FT, Hucka M, Kolpakov F, Andrew KM, et al. Reproducible computational biology experiments with SED-ML - the simulation experiment description markup language. BMC Syst Biol 2011;5:198.

40. Bergmann FT, Adams R, Moodie S, Cooper J, Glont M, Golebiewski M, et al. COMBINE archive and OMEX format: one file to share all information to reproduce a modeling project. BMC Bioinf 2014;15:369.

41. Bergmann FT, Rodriguez N, Le Novère N. COMBINE archive specification version 1. J Integr Bioinform 2015;12:261.

42. Gennari JH, König M, Misirli G, Neal ML, Nickerson DP. OMEX metadata specification version 1.2. J Integr Bioinform 2021;18:2021-0020.

43. Li C, Courtot M, Le Novère N, Laibe C. Biomodels. net web services, a free and integrated toolkit for computational modelling software. Briefings Bioinf 2010;11:270-7.

44. BioModels.net Qualifiers. 2020. Available from: http://co.mbine.org/standards/qualifiers.

45. Juty N, Le Novère N, Laibe C. Identifiers. org and miriam registry: community resources to provide persistent identification. Nucleic Acids Res 2012;40:D580-6.

46. Identifiers.org. 2020. Available from: http://identifiers.org/.

47. Courtot M, Juty N, Knüpfer C, Waltemath D, Zhukova A, Dräger A, et al. Controlled vocabularies and semantics in systems biology. Mol Syst Biol 2011;7:543.

48. Systems biology ontology on BioPortal. 2019. Available from: http://bioportal.bioontology.org/ontologies/SBO.

49. Kinetic simulation algorithm ontology on BioPortal. 2021. Available from: http://bioportal.bioontology.org/ontologies/ KISAO. 
50. Neal ML, König M, Nickerson D, Mısırlı G, Kalbasi R, Dräger A, et al. Harmonizing semantic annotations for computational models in biology. Briefings Bioinf 2019;20:540-50.

51. Neal ML, Gennari JH, Waltemath D, Nickerson DP, König M. OMEX metadata specification version 1.0. J Integr Bioinform 2020;17:20.

Supplementary Material: The online version of this article offers supplementary material (https://doi.org/10.1515/jib-20210026). 